\title{
Should Knowlesi Malaria In Southeast Asia Impose A Global Health Alert?
}

\section{Sergio Sabbatani ${ }^{1}$, Sirio Fiorino ${ }^{2}$, Elisabetta Chili $^{2}$ and Roberto Manfredi ${ }^{1}$}

${ }^{1}$ Infectious Diseases, University of Bologna, S. Orsola-Malpighi Hospital, Bologna, Italy

${ }^{2}$ Internal Medicine, Budrio General Hospital, Budrio, Italy

Keywords: Malaria parasites; Plasmodium knowlesi; Emerging plasmodia; Human health; Public health concerns

\section{Introduction}

Four main malaria plasmodia involve human beings. Plasmodium vivax, Plasmodium malariae and Plasmodium ovale had an older evolution paralelling the human species evolution (co-evolution) [1-3], while Plasmodium falciparum has been encountered by humans later during their evolution, since it was acquired by monkeys probably between the end of Mesolithic and the beginning of Neolithic eras [4]. Over twenty species of malaria plasmodia are capable of infecting monkeys (Table 1), but the anecdotal transmission of these organisms to humans has been considered until recently a rare event, with minor public health concerns. The transmission of these plasmodia protozoa predominantly occurred in South-East Asia, China, and CentralSouthern America [5]. The expansion of human activities in geographic areas which remained poorly anthropized in the past (i.e. forest zones where productive activities have been recently started), and wild regions, which are increasingly visited by travellers for eco tourism activities, represent objective elements which lead to an expected increase of risk to acquire malaria plasmodia of simian origin. Moreover, the present demographic expansion of local populations in different regions of the Southern Asia and Southern America, which also involved wild areas close to rain forests, especially when villagers carrying out agricultural or breeding activities are interested, bear further epidemiological risk factors. In these environmental contexts, an overlapping of human and simian habitats becomes evident, in presence of a common infestation by Anopheles mosquitoes, which are the needed vehicle for malaria transmission to humans. Among the twenty plasmodia species which may infect monkeys, five of them have been documented as potential infectious agents for humans: they are Plasmodium simium, Plasmodium brasilianum (found in South America), Plasmodium cynomolgi, Plasmodium inui, and Plasmodium knowlesi (retrieved in South-Eastern Asia) [5]. The majority of malaria infection of monkeys tend to cause a mild-to-moderate disease in humans, which is frequently self-limiting, and rarely requires antimalaric chemotherapy, since they are often missed or neglected. The identification of $P$. knowlesi disease foci confirmed by biomolecular techniques in Sarawak (Malaysian Boneo), Sabah (a state belonging to the Malaysia Confederation), and Pahang (peninsular Malaysia) between the end of the past century and early XXI century [6,7], caught the attention of researchers and clinicians operating in South-Eastern Asia, and more recently of the international health care authorities [5], initially focusing the attention on episodes which presented a typical malaria course, but received a microscopic diagnosis of $P$. malariae infection. A distinctive feature of these patients was the elevated parasitemia and the severity of clinical course, should a rapid and appropriate treatment was not administered [6,7]; in the records of these authors also four cases with a lethal evolution were present. Later, the availability of specific $P$. knowlesi primers allowed to pose a definitive diagnosis also in subjects whose erythrocytes proved morphologically similar to those infected by $P$. malariae, but tested negative when assessed with $P$. malariae primers. The more severe impact of P. knowlesi malaria is related to the evidence that this Plasmodium has a daily replication cycle and may therefore reach an elevated, life-threatening parasitemia in absence of an effective treatment, whereas $P$. malariae has a replication cycle every three days (so-called quartan fever), and never reaches elevated blood parasite concentrations [6-8]. Recent entomological studies [9] demonstrated that in the Malaysian geographic context the more efficient vector transmitting $P$. knowlesi infection to humans is Anopheles latens $[10,11]$ and highest biting rate is registered at the limits of forest $(6.74 \%)$, while a reduce frequency regards the rain forest $(1.85 \%)$ and the examined longhouses (the typical Malaysia houses) $(0.28 \%)$, which never were the site of micro-epidemic disease clusters. Based on these data, it has been hypothesized that humans acquire infection in the forest, when hunting, or returning home at dusk. [10,11]. The potential simian reservoirs are Macaca mulatta, M. nemestina, M. inus, and Saimiri sciurea. After the report of Malaysia foci, where native population was involved, and the identification of single imported cases in Europe and North America by tourists who travelled for short periods close to forest areas of South-Eastern Asia [12-16], the attention of researchers for $P$. knowlesi rapidly increased. In the year 2008, the Journal "Nature" published the analysis of the nuclear genomic sequencing of $P$. knowlesi [17]. It was the first complete gene sequencing of a simian Plasmodium, therefore offering the opportunity for a comparation with the complete genome of $P$. vivax and other already known Plasmodia genomes. In contrast with other plasmodial genomes, putative familiar antigenic variants are scattered throughout the genome and associated with intrachromosomal telomere repeats. One of these families, called KIRs, includes genomic sequences which on the whole match around one-half of the extracellular domain CD99 of the host: this situation suggested that this form could represent an unusual molecular mimicry $[17,18]$. A relevant information came from recent observations and epidemiological data came from Malaysia. The habitat of humans is more and more overlapping that of primates, and it is primarily due to two aspects: the first is related to the expansion of human activities (agriculture, deforesting, clearing of valued wood, and animal breeding), in areas also inhabitated by monkeys. The second emerging issue is related to the relevant increase of touristic activities (i.e. trekking) by international visitors, who spend a proportionally limited time in this environment, and subsequently go back to their countries of origin [8]. In this last situation, the limited knowledge of

${ }^{*}$ Corresponding author: Dr. Sergio Sabbatani, Infectious Diseases, S. Orsola Hospital Via Massarenti 11 I-40138 Bologna, Italy, Tel: +39-051-6363355; Telefax: +39-051-343500; E-mail: Sergio.sabbatani@aosp.bo.it

Received May 06, 2011; Accepted November 10, 2011; Published November 15, 2011

Citation: Sabbatani S, Fiorino S, Chili E, Manfredi R (2011) Should Knowlesi Malaria In Southeast Asia Impose A Global Health Alert?. J Bacteriol Parasitol S2 002. doi:10.4172/2155-9597.S2-002

Copyright: (c) 2011 Sabbatani S, et al. This is an open-access article distributed under the terms of the Creative Commons Attribution License, which permits unrestricted use, distribution, and reproduction in any medium, provided the original author and source are credited. 
Citation: Sabbatani S, Fiorino S, Chili E, Manfredi R (2011) Should Knowlesi Malaria In Southeast Asia Impose A Global Health Alert?. J Bacteriol Parasitol S2-002. doi:10.4172/2155-9597.S2-002

Page 2 of 6

\begin{tabular}{|c|c|c|}
\hline Simian Plasmodium species & Regional distribution & Human species resembling to them \\
\hline \multicolumn{3}{|l|}{ Asia } \\
\hline P. coatney & Malaysia, Philippines & P. falciparum \\
\hline P. cynomolgi & India, Indonesia, Malaysia, Sri Lanka, Taiwan & P. vivax \\
\hline P. eylesi & Malaysia & P. vivax \\
\hline P. fieldi & Malaysia & P. ovale \\
\hline P. fragile & India, Sri Lanka & P. falciparum \\
\hline P. hylobati & Indonesia & P. vivax \\
\hline P. inui & India, Indonesia, Malaysia, Philippines, Sri Lanka, Taiwan & P. malariae \\
\hline P. jeffrey & Indonesia, Malaysia & P. vivax \\
\hline P. knowlesi & China, Indonesia, Malaysia, Philippines, Singapore, Thailand, Taiwan & P. malariae, P. falciparum \\
\hline P. pitheci & Malaysia & P. vivax \\
\hline P. simiovale & Sri Lanka & P. ovale \\
\hline P. silvaticum & Malaysia & P. vivax \\
\hline P. youngi & Malaysia & P. vivax \\
\hline \multicolumn{3}{|l|}{ South America } \\
\hline$P$. brasilianum & Brazil, Colombia, Mexico, Panama, Peru, Venezuela & P. malariae \\
\hline P. simium & Brazil & P. vivax \\
\hline
\end{tabular}

Table 1: Malaria species of simian origin isolated in Asia and in Southern America (modified from the reference quotation [4]). Their associated geographical distrubution, and the morphologic similarities to one of the four "classical" human plasmodia species (i.e. Plasmodium falciparum, Plasmodium vivax, Plasmodium ovale, and Plasmodium malariae), are pointed out.

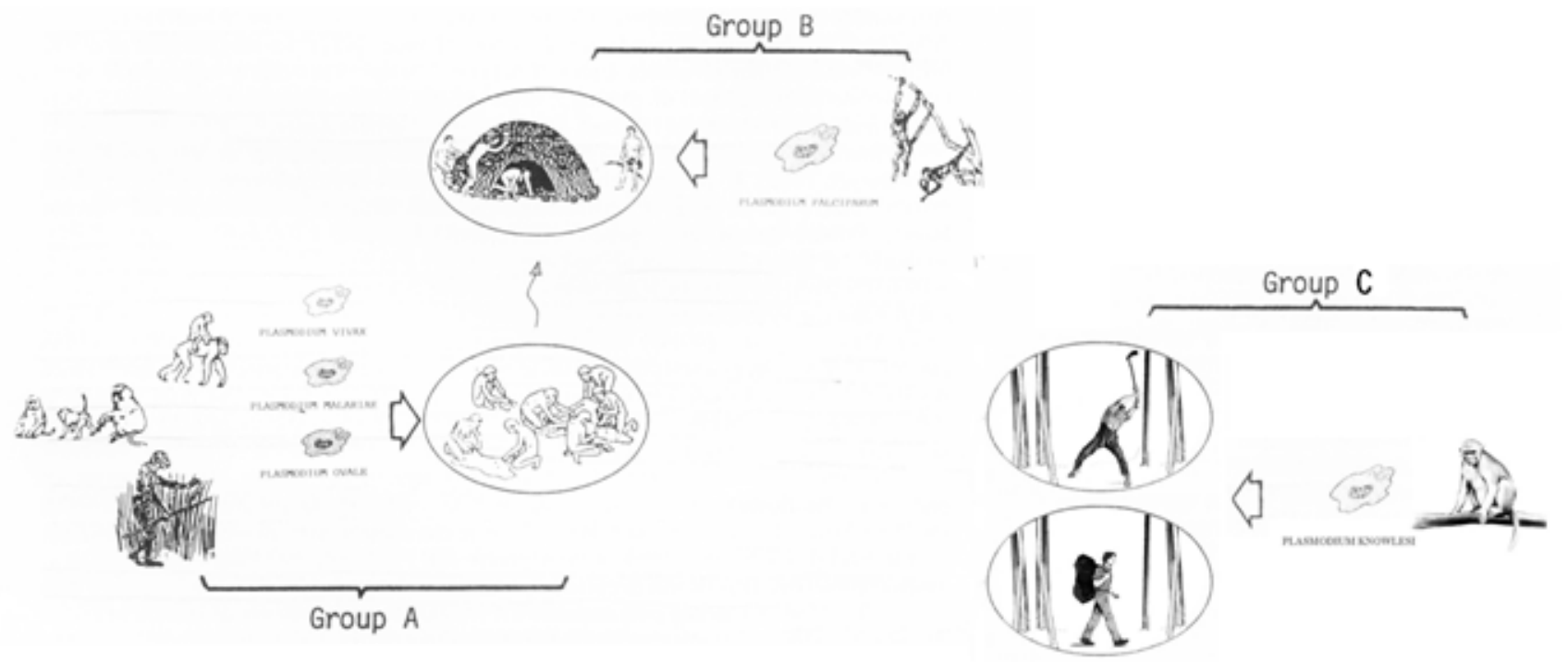

Figure 1: The enclosed graph depicts the biological and filogenetical evolutions of $P$. vivax, $P$. malariae, and $P$. ovale (Group A), compared with the extremely more recent evolution pathway of $P$. falciparum (group B). $P$. knowlesi (belonging to group C), is the last Plasmodium species which steadily entered the human pathology, and is related to productive and touristic human activities carried out in the forest regions of Malaysia and Borneo. The image has been taken and modified from some studies published by L. Capasso (quoted at reference [1]), in particular with the adjunct of group C malaria parasite.

simian plasmodia and thir diagnostic-identification problems, may confuse Western health care providers and all physicians working in non-endemic areas of the entire world. This may occur especially when a basic microscopic, parasitologic examination is carried out for the research and identification of malaria plasmodia, since this technique does not ensure to distinguish P. malariae from P. knowlesi, therefore posing at risk patient's life [8]. The biomolecular techniques are extremely useful to trace the general epidemiology of malaria, and to clarify the role and importance of mixed infections, which still remain underestimated [8]. Unfortunately these sophisticated assays are costly and not easily available throughout the world, especially in developing countries, while the next steps in the development of a standardized, sensitive and specific diagnosis of $P$. knowlesi infection should be based just on standardized molecular biology techniques On the basis of epidemiological features of regions where epidemic foci of $P$. knowles $i$ malaria have been reported, and the anecdotal episodes of clinical reports occurred in Western travellers coming back to their countries of origin with a P. knowlesi disease, it is not possible to establish whether infections have been acquired by the most common animal reservoirs (i.e. macaques), or whether human-to-human contagion may have occurred. However, it is presently clear that this zoonosis is no more responsible of single, episodic cases, but it represents a true health care emegency in South-Eastern Asian countries, where multiple, successful efforts to contain malaria as a whole have been already performed Figure 1 allows to appreciate the overall co-evolutive pathway of malaria plasmodia in parallel with that of the genus Homo, 
from hominids (group A), toward modern men (group C), with the appearance of $P$. falciparum which entered the epidemiological scenario infecting humans (group B) of mesolithic and early neolithic eras, who spent their lives in cabins located at the edge of rain forest and in the proximity of the first cultured fields. Just the introduction of the first organized human activities which significantly contributed to the expansion of $P$. falciparum, which up to date represents the most relevant malaria pathogen from the pathocenosis of pre-historical populations, unavoidably conditioning both economic and anthropological development. With regard to the appearance of a novel malaria parasite epidemiologically relevant for humans, the hope is that this novel patocenosis remain circumscribed in some countries of South-Eastern Asia, without extension to other areas, which are susceptible due to similar climatic and environmental features. In this evenience, the impact of autochtonous populations, not naturally immunized, after the contacts which normally occur during early infancy, may be responsible for particularly severe consequences, not only for local populations lacking of a specific immune memory and exposed to a novel human Plasmodium species, but also for the growing number of tourists, who may be exposed to the risk to come back to their countries of origin with a $P$. knowlesi malaria infection, whose diagnosis cannot rely on the routine laboratory examinations.

\section{References}

1. Capasso L (1985) L'origine delle malattie. M. Solfanelli, Chieti, Italy.

2. Kim KC (1985) Coevolution of Parasitic Arthropods and Mammals. John Wiley \& Sons, New York.

3. Cockburn A (1963) The Evolution and Eradication of Infections Diseases. Greenwood Press, Westport.

4. Cohen MN, Armelagos GJ (1984) Paleopathology at the Originis of Agriculture. Academic Press Inc., Orlando.

5. Centers for Disease Control and Prevention (CDC) (2009) Simian malaria in a U.S. traveler--New York, 2008. MMWR Morb Mortal Wkly Rep 58: 229-232.
6. Singh B, Kim Sung L, Matusop A, Radhakrishnan A, Shamsul SS, et al. (2004) A large focus of naturally acquired Plasmodium knowlesi infections in human beings. Lancet 363: 1017-1024.

7. Cox-Singh J, Davis TM, Lee KS, Shamsul SS, Matusop A, et al. (2008) Plasmodium knowlesi malaria in humans is widely distributed and potentially lifre threatening. Clin Infect Dis 46: 165-171

8. White NJ (2008) Plasmodium knowlesi: the fifth human malaria parasite. Clin Infect Dis 46:172-173.

9. Vythilingam I, Tan $\mathrm{CH}$, Asmad M, Chan ST, Lee KS, et al (2006) Natura transmission of Plasmodium knowlesi to humans by Anopheles latens in Sarawak, Malaysia. Trans R Soc Trop Med Hyg 100: 1087-1088.

10. Tan CH, Vythilingam I, Matusop A, Chan ST, Singh B (2008) Bionomics of Anopheles latens in Kapit, Sarawak, Malaysian Borneo in relation to the transmission of zoonotic simian malaria parasite Plasmodium knowlesi. Malaria J 7: 52.

11. Vythilingam I (2010) Plasmodium knowlesi in humans: a review on the role of its vectors in Malaysia. Trop Biomed 27: 1-12.

12. Kantele A, Marti H, Felger I, Müller D, Jokiranta TS (2008) Monkey malaria in an European traveller returning from Malaysia. Emerg Infect Dis 14: 14341436.

13. Jongwutiwes S, Putaporntip C, Iwasatit T, Sata T, Kambara H (2004) Naturally acquired Plasmodium knowlesi malaria in human, Thailand. Emerg Infect Dis 10: $2211-2213$

14. Zhu HM, Li J, Zheng H (2006) Human natural infection of Plasmodium knowlesi. Zhongguo Ji Sheng Chong Xue Yu Ji Sheng Chong Bing Za Zhi 24: 70-71.

15. Ng OT, Ooi EE, Lee CC, Lee PJ, Ng LC, et al. (2008) Naturally acquired human Plasmodium knowlesi infection, Singapore. Emerg Infect Dis 14: 814-816.

16. Bronner V, Divis PC, Färnert A, Singh B (2009) Swedish traveller with Plasmodium knowlesi malaria after visiting Malaysian Borneo. Malar J 8: 15

17. Carlton JM, Adams JH, Silva JC, Bidwell SL, Lorenzi H, et al. (2008) Comparative genomics of the neglected human parasite Plasmodium vivax. Nature. 455: 757-763.

18. Pain A, Böhme U, Berry AE, Mungall K, Finn RD, et al. (2008) The genome of the simian and human malaria parasite Plasmodium knowlesi. Nature 455 799-803.
This article was originally published in a special issue, COPD: Epidemiology and New Therapeutics handled by Editor(s). Dr. A.B.Raja Chatterjee, Wake Forest University, USA 